\title{
RELATIVISTIC FILAMENTATION INSTABILITY IN AN ARBITRARILY ORIENTED MAGNETIC FIELD
}

\author{
E. Pérez-Álvaro ${ }^{1}$ and A. Bret ${ }^{1}$
}

\begin{abstract}
Although high-energy cosmic rays (HECRs) and gamma-ray bursts (GRBs) are the most energetic phenomena occurring in the universe, their origin are important enigmas in the field of astrophysics. Today, the most studied scenario that attempts to explain them is known as the Fireball Model. This theory assumes that the particles are accelerated by a shock developing in the interior of a relativistic plasma from a supernova (SN). The filamentation (sometimes called "Weibel") instability is believed to mediate collisionless shock formation from the collision of two plasma shells. It has been known for long that a flow aligned magnetic field can completely cancel this instability. In this work, we analyze the robustness of the filamentation instability which develops inside a plasma immersed in an arbitrarily oriented magnetic field.
\end{abstract}

\section{Introduction}

According to the Fireball Model, the GRBs generation mechanism is obtained when a central source releases a large amount of fluid consisting of electrons, positrons and in some cases contaminated by baryons (protons) in a very short time in a very small region (about $10^{12} \mathrm{~cm}$ ). The plasma moving at relativistic speed is what is called Fireball. Inside the Fireball, shocks can develop in which the particles can be accelerated by means of electromagnetic interaction Narayan (1992). These shocks are generated when internal layers of the fireball come into contact. Particles accelerated by the shock begin to emit synchrotron radiation. In the Fireball, electromagnetic instabilities appear to be important for the development of electromagnetic shock. This phenomenon has been studied with numerical simulations Silva (2003) and it has been verified that the filamentation instability (which $k$ is normal to the flow), is responsible for the shock development.

1 ETSI Industriales, University of Castilla-La Mancha, Institute of Energetics Investigations and Industrial Applications, 13071 Ciudad Real, Spain 
Within a magnetized medium, nearly all works consider a magnetic field $\mathbf{B}_{0}$ parallel to the movement, even though in real conditions, a magnetic field is not perfectly aligned. In the case of the collision of two plasmas in a magnetic field parallel to the direction of propagation, the filamentation instability is canceled from a critical value of the field Godfrey (1975). At this point, we want to investigate the development of the filamentation instability immersed in a magnetic field oriented arbitrarily in order to determine whether such suppression of this instability persists Bret \& Perez Alvaro (2011).

\section{Formalism and dispersion equation}

We consider two identical electron-proton plasmas, with density $n$ and traveling along the same direction of motion $(z$ axis) but in opposite directions with same speed $\boldsymbol{V}_{0}$ and corresponding Lorentz factor $\gamma_{0}=\left(1-\boldsymbol{V}_{0}^{2} / c^{2}\right)^{-1 / 2}$. Being $\theta$ the angle between the magnetic field $\boldsymbol{B}_{0}$ and the flow, the magnetic field is defined as $\boldsymbol{B}_{0}=B_{0} \sin (\theta) \boldsymbol{u}_{x}+B_{0} \cos (\theta) \boldsymbol{u}_{z}$. Since the mass of the proton is much greater than that of the electrons and the Lorentz factor is the same for both, we consider proton mass infinite.

We work in the cold fluid approximation and therefore the conservation and momentum equations for electrons are given by:

$$
\begin{gathered}
\frac{\partial n}{\partial t}+\nabla \cdot(n \boldsymbol{V})=0 \\
\frac{\partial \boldsymbol{p}}{\partial t}+(\boldsymbol{V} \cdot \nabla) \boldsymbol{p}=q\left[\boldsymbol{E}+\frac{\boldsymbol{V} \times\left(\boldsymbol{B}+\boldsymbol{B}_{0}\right)}{c}\right]
\end{gathered}
$$

where $\boldsymbol{p}=\gamma m \boldsymbol{V}, q$ and $m$ are the momentum, charge and mass of the electron respectively. These equations were linearized thanks to a standard process Bret \& Perez Alvaro (2011) and introduced in the expression for de current $\boldsymbol{J}_{1}=$ $q \sum_{j=1,2}\left(n_{j 0} \boldsymbol{V}_{j 1}+n_{j 1} \boldsymbol{V}_{j 0}\right)$ where $j=1,2$ represents each plasma. In the same way the Maxwell-Faraday equation is linearized and introduced in the MaxwellAmpere equation, to obtain:

$$
\frac{c^{2}}{\omega^{2}} k \times\left(\boldsymbol{k} \times \boldsymbol{E}_{1}\right)+\boldsymbol{E}_{1}+\frac{4 i \pi}{\omega} \boldsymbol{J}_{1}=0 .
$$

Equation (2.3) is tensorial of the form $T\left(\boldsymbol{E}_{1}\right)=0$. The dispersion equation was obtained from $\operatorname{det}(T)=0$ Bret \& Perez Alvaro (2011). In addition, we used the dimensionless parameters $Z=\frac{k V_{0}}{\omega_{p}}, x=\frac{\omega}{\omega_{p}}, \beta=\frac{V_{0}}{c}$ and $\Omega_{B}=\frac{\omega_{b}}{\omega_{p}}$ where $\omega_{p}^{2}=$ $\frac{4 \pi n q^{2}}{m}$ is the electronic plasma frequency and $\omega_{b}=\frac{|q| \boldsymbol{B}_{0}}{m c}$ the cyclotron frequency.

\section{Results and analytical expressions}

Filamentation instability grows exponentially like $e^{\delta t}$, where $\delta$ is the growth rate which is obtained by solving the dispersion equation $\operatorname{det}(T)=0$, and taking the 
imaginary part of the solution $\omega$. To analyze the results, we observed the dependence of $\delta$ with the magnetic field parameter $\Omega_{B}$ and the reduced wave vector $Z$ in different three-dimensional representations that were obtained for angles $\theta$ between $\left[0, \frac{\pi}{2}\right]$. Some examples for these $3 \mathrm{D}$ representations can be seen in Figure 1.

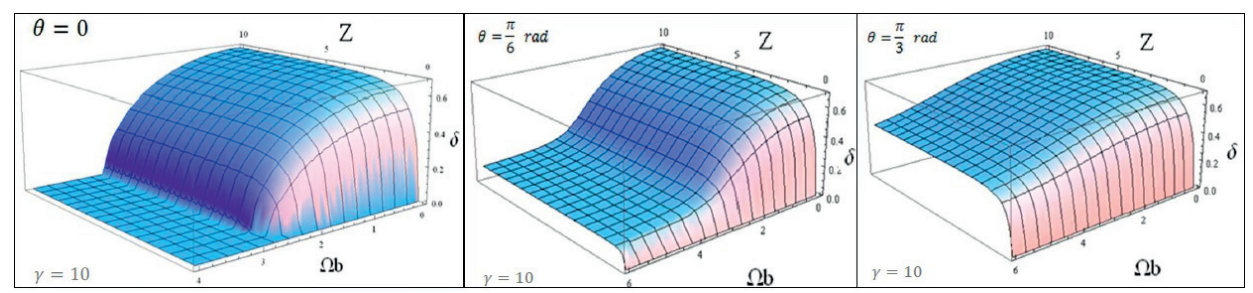

Fig. 1. Growth rate $\delta$ as a function for the magnetic field parameter $\Omega_{B}$ and reduced wave vector $Z$, with $\gamma=10$. Left to right $\theta=0, \theta=\frac{\pi}{6}$ and $\theta=\frac{\pi}{3}$.

Analyzing these representations, we notice the growth rate reaches a maximum for $Z \rightarrow \infty$, and compute the dispersion equation in this limit. This maximum growth rate can be evaluated analytically and reads $\delta^{*}$ :

$$
\delta^{*}=\sqrt{\frac{2 \beta^{2} \sin ^{2}(\theta)}{\gamma^{3} \cos ^{2}(\theta)+\gamma \sin ^{2}(\theta)}} .
$$

Using a Taylor expansion in the expression of the growth rate for weak magnetization, we obtain the value of the parameter of the critical field $\Omega_{B}^{*}$ beyond which the growth rate saturates:

$$
\Omega_{B}^{*}=\frac{2 \beta \sqrt{\gamma}}{\cos (\theta)} .
$$

\section{Conclusion}

These results were obtained under various assumptions: cold plasmas, protons with infinite mass and identical plasmas. The Fireball model attempts to explain the origin of HECRs and GRBs assuming a shock develops within a relativistic plasma from a supernova can accelerate particles. Without the Filamentation instability the shock would not develop. We show in this paper that the filamentation instability can be canceled only if the motion of plasma and the magnetic field are fully aligned.

\section{References}

Bret, A., \& Pérez-Álvaro, E., 2011, Phys. Plasma, 18, 080706

Godfrey, B.B., Shanahan, W.R., \& Thode, L.E., 1975, Phys. Fluids, 18, 346

Narayan, R., Paczynski, B., \& Piran, T., 1992, ApJ, 395, L83

Silva, L.O., Fonseca, R., Tonge, J.W., et al., 2003, ApJ, 596, L121 
